ORIGINAL ARTICLE

\title{
Sensitivity of Plasma Micrornas as a Probable Non-Small Cell Lung Cancer Biomarker and Its Significance
}

\author{
QAZI TALAL ALI, MUHAMMAD JAVAID IQBAL, AYESHA MASOOD ${ }^{3}$, AAMIR HUSSAIN ${ }^{4}$, MUHAMMAD USMAN ${ }^{5}$ \\ ${ }^{1}$ Medical Officer, Al Maroof Hospital, Islamabad \\ ${ }^{2}$ Assistant Professor Pathology, Aziz Fatima Medical and Dental College, Faisalabad \\ ${ }^{3}$ Assistant Professor, Department of Pathology, UCMD, University of Lahore \\ ${ }^{4}$ Assistant Professor Medicine, Fazaia Ruth PFAU Medical College, Karachi \\ ${ }^{5}$ FCPS Hematology, Associate Professor of Pathology, Aziz Fatima Medical and Dental College, Faisalabad \\ Corresponding author: Dr. Qazi Talal Ali, Email: talalali7007@gmail.com,Cell No.+92-334-7107007
}

\begin{abstract}
Non-small cell lung cancer (NSCLC) is the chief origin of death due to cancer. The development of a less invasive diagnostic technique for NSCLC, especially at the beginning, can improve outcomes. By means of microarray platforms, we formerly diagnosed 12 microRNAs (miRNAs) abnormally expressed in primary cancer cells associated with early NSCLC.

Objective: In this study, we will extend previous studies to determine if miRNAs may be beneficial as a NSCLC potential plasma biomarker.

Methods: We primarily confirmed miRNA expression from PCR of 32 stage one NSCLC patients with lung tumor and plasma specimens were assessed, then assessed the investigative value of plasma miRNAs in 61 patients of NSCLC and 30 normal subjects. It was confirmed that the alteration of MiRNA expression influences the regeneration of neoplastic tumors. MiRNAs were steady and reliable in plasma measurements. The cohort consisted of 21 men and 11 women. Their age ranges from 47 to 80 years. AC tumors were classified in 17 and SCC in 15 patients. Smoking packets use in these patients were $39 \pm 28$. In addition, IV blood were taken from healthy subjects with matched data distribution to studied group as age, race, sex and smoking, and aided as a control to evaluate fluctuations in plasma in cancer patients.

Results: Five out of 12 miRNAs showed a significant change of level in plasma and the corresponding tumor tissue (all r40850, all $\mathrm{P}<0.05$ ). Four genes (miRNA-126, 21, 486-5 and 210p) set the best logistic regression pattern, with $87 \%$ sensitivity and $97 \%$ of specificity of at the time of NSCLC in differentiating from healthy patients. Moreover, the miRNA genes generated a sensitivity of $79.41 \%$ and $92.59 \%$ of specificity in patients diagnosed with SCC and AC. Moreover, genes have specificity of $96.67 \%$ in the analysis of lung adenocarcinoma and squamous cell carcinoma. (P less than 0.05 ). A change in square miRNA expression may offer potential NSCLC blood-derived biomarker.

Conclusion: Finally, we show that miRNA expression identified from surgical tumor tissue can be easily and correctly determined in plasma. Though, prominently, a plasma miRNA recognition panel would be cast-off as a less invasive analytic method for NSCLC, including long-term adenocarcinoma. However, the presence of an independent potential biomarker requires further verification.

Keywords: Lung cancer; Diagnosis; Plasma; Microrna; Qrt-PCR
\end{abstract}

\section{INTRODUCTION}

Non-small cell lung cancer (NSCLC) is the utmostcommunal lung cancer type and is the chief cause of mortality in the USA and around the world. NSCLC chiefly comprises of 2 main forms of histology: squamous cell carcinoma (SCC) and adenocarcinoma (AC). NSCLC is often detected in last stages, ensuing 14\% 5-year survival rate, approximately. In contrast, the 5-year endurance percentage of stage I NSCLC positive individuals receiving successful treatment may be up to maximum of $83 \%$. So, early detection of NSCLC can decrease death ratio ${ }^{1-3}$. Prompt recognition of NSCLC on X-ray and sputum cytology has less sensitivity. Bronchoscopy is great for detecting central occurring tumors of the lungs. Though, it's aninvasive procedure. Though computed tomography offers good anatomical information and can perceivenoninvasively small-size NSCLC, better sensitivity is associated with overdiagnosis ${ }^{4}$. Therefore, the development of minimally invasive techniques using the latest advances in molecular genetics is clinically important for the early diagnosis of NSCLC ${ }^{5}$. Blood plasma is obviously the best option for developing these diagnostic markers. Many molecular alterations of specific tumors are recognized in serum or plasma and have been revealed to be a biomarker in lung cancer patients ${ }^{6-7}$. However, none of the markers tested so far has achieved the features allowing for the NSCLC diagnosis. MicroRNAs (miRNAs) have revealed now discoveries in the molecular analysis of malignancy ${ }^{8}$. Proven studies reports that the blood circulating miRNAs, are helpful for detection of tumors including lung cancer, can be used as cancer biomarkers. E.g., recently, miRNA serum signatures have been recognized that can forecast the endurance of patients affected with NSCLC. While it is postulated that measuring miRNA expression in plasma / serum is a promising approach for the diagnosis of lungs tumor, the idea entails more research to validate its probable clinical benefit ${ }^{9-10}$. By means of microarray platforms, we newly recognized twelve miRNAs whom if abnormally expressed in human tumor tissues exhibited initial NSCLC staging ${ }^{11}$. So, now we extend this research based on previous studies by examining whether miRNAs can be found in plasma and cast-off as less invasive biomarkers of NSCLC. First, we established the miRNA findings in pairs of surgical tissues 
and specimens of plasma from independent cases of NSCLC by means of quantitative Real-time qRT-PCR. We then analyzed plasma miRNA expression in a cohort of 61 patients of NSCLC and 32 healthy individuals to evaluate the analyticalworth of NSCLC. Studies have shown that changes in plasma miRNA expression have the potential to provide blood-derived biomarkers for NSCLC ${ }^{12}$.

\section{MATERIAL AND METHODS}

There are two phases of this study. In the $1^{\text {st }}$ phase, we were all able to confirm whether the miRNAs changes are related with NSCLC stage I, the abnormal appearance of which could be detected in surgical independent tissues and whether the miRNAs change in the plasma matched appropriately with the equivalent tumors. So, we gained the paired tissues of lung tumor, plasma specimens and noncancerous lung tissues of 32stage I patients of NSCLC (Table 1).

Table 1 Shows 32 Stage-I NSCLC Cases Matched with 29 Healthy Subjects and Their Clinical and Characteristic Features

\begin{tabular}{|l|l|l|}
\hline \multicolumn{2}{|l|}{32 cases of NSCLC } & $\begin{array}{l}\text { 29 Healthy } \\
\text { smokers }\end{array}$ \\
\hline Age, median (s.d.) & $67(7.1)$ & $68.2(6.4)$ \\
\hline Gender & $11(34.4 \%)$ & $20(69 \%)$ \\
\hline Male & $21(65.6 \%)$ & $9(31 \%)$ \\
\hline Female & \multicolumn{2}{|l|}{} \\
\hline Status of Smoking & $36.4(24.2)$ & $36.9(26.8)$ \\
\hline $\begin{array}{l}\text { Pack per years, median } \\
\text { (s.d.) }\end{array}$ & \multicolumn{2}{|l|}{} \\
\hline Stage & $32(100 \%)$ & \\
\hline Stage I & $17(53.1 \%)$ & \\
\hline The Histological types & $15(46.9 \%)$ & \\
\hline Adenocarcinoma &
\end{tabular}

The study consisted of 21 men and 11 women. Their age ranges from 47 to 80 years. AC tumors were classified in 17 and SCC in 15 patients. The smoking packets use in these patients were $39 \pm 28$ per year. In addition, IV blood were taken from 29 healthy subjects with matched data distribution to studied group as age, race, sex and smoking, and aided as a control to evaluate fluctuations in plasma in cancer patients. The test was performed according to the study protocols. Blood samples from cancer patients were taken prior to anesthesia induction, prior to surgical procedure. No one was given adjuvant radiotherapy or chemotherapy preoperatively.

The $2^{\text {nd }}$ phase discusses the diagnostic worth of miRNAs in NSCLC plasma. Blood samples were obtained according to the international protocols. NSCLC samples were collected from 61patients of SCC and AC and 30 healthy people (Table 2 ) by NSCLC at various stages and histological types. Of the cancer patients,22 were female, and 39 were male.

A total of 14 have NSCLC stage Icancer, stage II in 17 patients, stage III in 14 subjects, and stage IV in 16 cases. The diagnosis was madehistologically after thoracotomy and bronchoscopic biopsy according to the WHO strategy. The classification of NSCLC phases is grounded on the WHO classification and International Staging of Lung Cancer System. Differences in race, age, smoking and gender between healthy control sand NSCLC patients was not significant. Peripheral blood $(10 \mathrm{ml})$ was withdrawn EDTA coated tubes. The cancer patients' blood was taken at the time of the primary consultation, prior to the final surgical treatment and / or adjuvant treatment. The samples were collected and processed at $1300 \times \mathrm{g}$ for two hours by centrifugation at four degree for 10 minutes. Plasma was relocated to a fresh tube and until use were preservedat $80^{\circ} \mathrm{C}$.

RNA was taken out from the tissue samples by means of the mirVana miRNA isolation kit. 1.33 ulof the solution of cDNA was augmented in twenty microliterper ml of the mixtures. PCR quantitative was accomplished on a Bio-Red IQ5 RT multi-color spot detection system. All assays were accomplished in triplicate, and two inter-plate controls and a no template control were achieved in each trial. The expression levels in MiRNA were considered using the comparative cycle threshold (CT) method. The fold variation was considered using theequation of $2 \Delta \Delta \mathrm{Ct}$.

Table II. Shows 61 NSCLC Cases Matched with 30 Healthy Subjects and Their Clinical and Characteristic Features

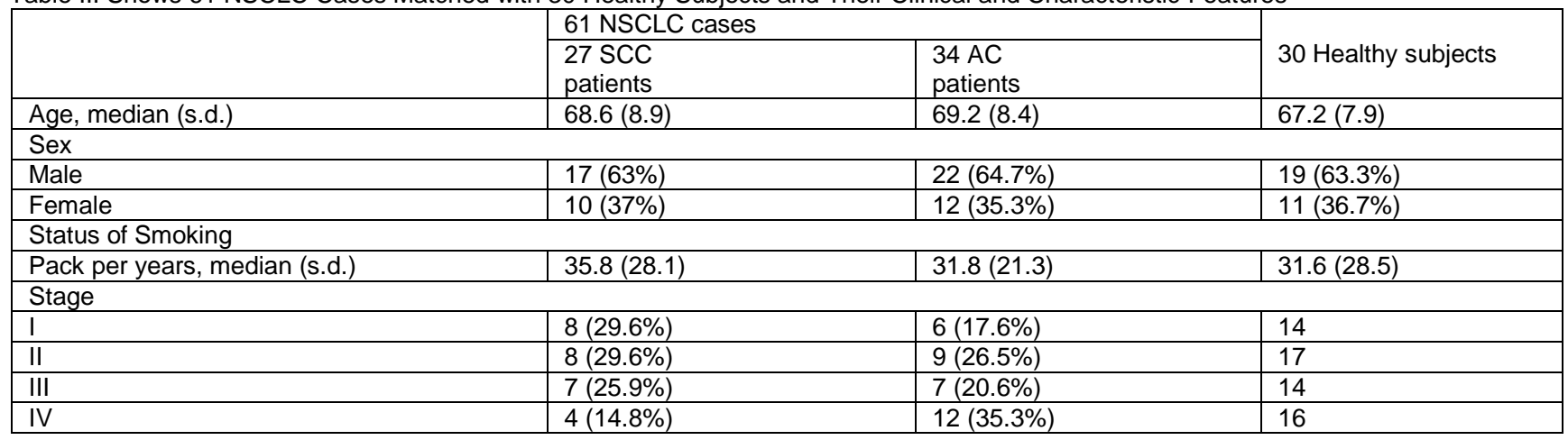


Sensitivity and Specificity of the qRT-PCR test for the MiRNAs quantification: Plasma was taken form 12healthy people and alienated into 4 portions of $350 \mathrm{ml}$. The $1^{\text {st }}$ aliquots of each sample were isolated on a day first for RNA isolation, the rest were stored at $20^{\circ} \mathrm{C}$ andmadeat $3^{\text {rd }}, 7^{\text {th }}$ and $30^{\text {th }}$ days. The $1^{\text {sttwo }}$ aliquots of every sample were incubated with different concentrations of RNase A (Qiagen). (0.1 andone $\mathrm{mg} / \mathrm{ml}$ ) for twenty hours correspondingly. One-third of all specimen were taken as control and used for treatment. Additionally, it was introduced into plasma treated samples with the synthesized miR238 RNase A cell (Qiagen) at various absorptions and without exemplary treatment. Endogenous miRNA expression and spiked miRNA expression were assessed in parallel samples to qRT-PCR, respectively. Furthermore, the plasma of the remaining healthy people was alienated into 3portions. The $1^{\text {st }}$ aliquots of every sample were repeated until freezing for 5 cycles, and the remaining 2 aliquots were kept in the refrigerator at $801 \mathrm{C}$ for three and seven days, correspondingly. The endogenous miRNAs expression in these samples was also calculated. To evaluate the accuracy of the QRT-PCR assay for quantifying miRNA in plasma, the CEL-Mir-238 in synthesized form was diluted in RNA isolated form, respectively, from 5denaturized samples of plasma. The primers preciseqRT-PCR to cel-miR-238 was achieved in the samples.Sensitive plasma samples with CEL-Mir-238 in synthesized form were tootried in parallel and cast-off as controls. The variants of miR-486 which were synthesizeddilute in DEPC water, followed by polyadenylation and reverse transcription. Independent qRT-Them reactions were performed for each Mir-486 variant whetherin each reaction there were specific PCR primers to solitary one of the two variations. To quantify plasma miRNAs to govern the dynamic range and sensitivity of qRTPCR, RNA was $1^{\text {st }}$ taken from the 5 healthy individuals' plasma and then dilute in DEPC water by 10 folds. Expression of twelve miRNAs was evaluated by qRT-PCR in consecutively samples in diluted form. All trials were tripled and repeated for 3 times.

Pearson's correlation analysis was used to evaluate the relationamid expression of tumor tissue in miRNA with plasma pairedspecimens. The examination was also used to evaluate the associationamid plasma miRNA expression and the patients'demographic characteristics and healthy subjects. Clinicopathologicalanalyses were taken as a reference standard to determine the specificity and sensitivity of gene therapy. The AUC analysis and receiver-operator characteristic curve was used with some precision to regulate each miRNA accuracy and to know each miRNA cut-off value in a particular sample and specificity percentage. The constrained parameters of Logistic regression models and comparable to minimum selection operator and absolute shrinkage were pragmatic to select an ideal potential biomarkers panel. All analyzes, counting the Wilcoxon's test, correlation coefficient, ANOVA, student text and logistic regression were achievedwithlog-transformed data. All values of the $\mathrm{P}$ are two sided and the less than 0.05 value of $P$ was also statistically significant.

\section{RESULTS}

Previously, microarray techniques were applied to examine primary lungs tumor and the expression variations we identified with 12 miRNAs were related with NSCLC stage I. MiRNAs are miRNAs-126, 21, 139, 145, 200b, 182, 210, 205, 375, 486-5p, 708 and 429. To govern whether miRNA abnormalities could be determined in tumor independent tissues we evaluatedqRT-PCR miRNA expression from 32 stage I NSCLC tissues and comparison was made with the non-cancerous tissue. All MiRNAs showed double or less tumor tissue expression compared to the corresponding non-carcinogenic substances (all after 0.001) (Table 3).

Table III shows 32 Stage-I NSCLC cases and their MiRNAs differentially expressed versus noncancerous paired specimens of lung tissue by qRT-PCR

\begin{tabular}{|l|l|l|}
\hline miRNAs & Log2 (fold) ${ }^{\star}$ (mean \pm s.d.) & P-value \\
\hline miR-126 & $2.86785679 \pm 0.24785434$ & 0.00028423 \\
\hline miR-21 & $2.68376890 \pm 0.27980678$ & 0.00072867 \\
\hline miR-139 & $2.14678230 \pm 0.20696445$ & 0.00015965 \\
\hline miR-145 & $2.47896724 \pm 0.21985626$ & 0.00052779 \\
\hline miR-205 & $2.87982563 \pm 0.26758932$ & 0.00058936 \\
\hline miR-182 & $2.22678450 \pm 0.28975487$ & 0.00027005 \\
\hline miR-210 & $2.31678590 \pm 0.31235786$ & 0.00076259 \\
\hline miR-200b & $2.16779289 \pm 0.24567893$ & 0.00032789 \\
\hline miR-429 & $2.01045975 \pm 0.31143657$ & 0.00023868 \\
\hline miR-375 & $2.85620858 \pm 0.26895849$ & 0.00057949 \\
\hline miR-708 & $2.27832248 \pm 0.32256860$ & 0.00055094 \\
\hline miR-486-5p & $2.86749864 \pm 0.28656724$ & 0.00043332 \\
\hline
\end{tabular}

To discover an internal control that can measure reliable miRNAs plasma expression, we assessed $C_{t}$ qRTPCR values of RNU6B and miR16 in all NSCLCs in plasma of healthy individuals. Both genes have formerly remained anticipated as a potential control for the internal normalization of quantitative miRNAs. To standardize the variance in experimental performance between various samples, all qRT sequences are derived from a given volume and the equal RNA amount or a fixed RNA volume ofinitial plasma $(350 \mathrm{ml})$ was taken. Additionally, we compare the $\mathrm{C}_{t}$ endogenous RNU6B and Mir-16 values with thespiked cel-miR-238. There was no significant difference in cancer patients in newly ready $\mathrm{C}_{t}$ plasma for CT RNU6B $(P=0.418)$ and Mir-16 $(P=0.464)$ values.

Table IV Shows Altered miRNA Expressions Prevalence in The Plasma Samples and Paired Tumor from 32 Stage-I NSCLC Cases

\begin{tabular}{|l|l|l|}
\hline \multirow{3}{*}{ miRNAs } & $\begin{array}{l}\text { The number of individuals } \\
\text { with transformed expressions } \\
\text { of miRNA (\%) }\end{array}$ & \\
\cline { 2 - 3 } & Positive cases of Tumor, (\%) & $\begin{array}{l}\text { Positive cases } \\
\text { in Plasma (\%) }\end{array}$ \\
\hline miR-182 & $30 / 32,(93.75)$ & $21 / 32,(65.63)$ \\
\hline miR-126 & $32 / 32,(100)$ & $22 / 32,(68.75)$ \\
\hline miR-486-5p & $32 / 32,(100)$ & $24 / 32,(75.00)$ \\
\hline miR-210 & $29 / 32,(90.63)$ & $20 / 32,(62.50)$ \\
\hline miR-21 & $31 / 32,(96.88)$ & $23 / 32,(71.88)$ \\
\hline
\end{tabular}

NSCLC-associated miRNA expression was reliably measured in plasma: To govern if there were twelve miRNAs in the plasma, we evaluatedits expression in the plasma of 12 healthy persons done by qRT-PCR. The total 
miRNAs tested had less than 32Ctvalues in every sample, representing that the miRNAs were in the plasma and can bedemonstrable. We then promoted the endogenous miRNAs stability in archival samples of plasma. The plasma samples aliquots were stowed for one, three, seven and thirty days at $20^{\circ} \mathrm{C}$. The bioanalystexhibited that the total RNA gradually decreased with the increase in $18 \mathrm{~S}$ and $28 \mathrm{~S}$ peaks from day $1^{\text {st }}$ to $30^{\text {th }}$ day.

Though, no effect on the miRNA expression level was observed, which was confirmed by qRT-PCR in the similar sample.

Since plasma had anincrease level of RNase activity, we measured miRNA expression treated in the samples with or without RNase A. To evaluate the accuracy of the quantification qRT-PCR of the plasma miRNAs, total RNA was mixed with synthesized cel-miR-238 from plasma denatured specimens. In mixed samples; Cel-Mir-238 was detected. Though, target miR-238 was not visible in RNA of humans as it was not spiked with the miRNA in synthesized form. In addition, there was exceptional linearity amid the qRT-PCR and the cel-mir-238 C values $\mathrm{Ct}_{t}$ values. Nonspecific intensification was noted, whereas only augmentation of the suitable gene therapy corresponding to the particular primer was detected. The findings suggest that $\mathrm{qRT}$ reverse transcriptase analysis is relatively accurate and can distinguish between miRNAs from the identical gene family. Consequently, miRNAs and plasma can be accurately detected.

The MiRNA panel has been optimized as a plasma biomarker for NSCLC: The 4 miRNAs used in mixture had high analytical sensitivity in last phases (II-IV) compared to NSCLC in stage I (P less than 0.05) (Table 5). Though, combination of the 4 genes resulted in a sensitivity of $73.33 \%$ and a sensitivity of $96.55 \%$. Differentiating NSCLC patients from healthy. Moreover, the the genes diagnostic sensitivity used collectively was higher significantly than that of $A C(91.67 \%)$ and SCC (82.35\%) (P less than 0.05$)$. Lastly, no connotation with smoking-related changes by age, gender, ethnicity among participants was noted.

Table $\mathrm{V}$ Shows the Diagnostic Accuracy of MiRNAs among NSCLC Patients

\begin{tabular}{|l|l|l|}
\hline & Sensitivity, $\%$ & Specificity, \% \\
\hline All cases & $86.89(53 / 61)$ & $96.67(29 / 30)$ \\
\hline Various histological types & & \\
\hline Squamous cell carcinoma & $79.41(27 / 34)$ & $96.67(29 / 30)$ \\
\hline Adenocarcinoma & $92.59(25 / 27)$ & $96.67(29 / 30)$ \\
\hline Cases with different stages & & \\
\hline I & $71.43(10 / 14)$ & $96.67(29 / 30)$ \\
\hline II & $88.24(15 / 17)$ & $96.67(29 / 30)$ \\
\hline III & $85.71(12 / 14)$ & $96.67(29 / 30)$ \\
\hline IV & $87.50(14 / 16)$ & $96.67(29 / 30)$ \\
\hline
\end{tabular}

\section{DISCUSSION}

In this analysis, we authenticated formerly recognized NSCLC by means of miRNAs associated with independent tumor tissues by qRT-PCR analysis. We also show that miRNAs were stable and easily measured in plasma ${ }^{13}$. Finally, we showed that the plasma miRNA panel offers probable biomarker that can distinguish healthy individuals from NSCLC cases. Elevation of MiR-21 observed in many human neoplasms ${ }^{14}$. Mir-21 has recently been classified as Oncomir and high levels may result in tumor progression and development. In addition, measuring plasma expression of miR-21 may be beneficial in the analysis of pancreatic cancer ${ }^{15}$. Moreover, high serum levels of miRNA-21 may be a predictor of the docetaxel-based chemotherapy efficacy in patients with metastatic hormonalmalignancy ${ }^{16}$. Furthermore, much upsurgerelationship in the level of miRNA expression was found amongsix miRNAs panel, counting miR-21, amid paired sera and breast tumors ${ }^{17-18}$. Moreover, miR-21 was suggestively excessive in normal controls than in cancer patients. Mir-21 may be the blood-derived biomarkers for the analysis, classification and diagnosis of breast cancer $^{19}$. Our research data suggests that miR-21 plasma may assist as a lung cancer biomarker for its diagnosis.Mir$486-5 p$ is rare in 8forms of human cancers, counting lung cancer. The opinion of plasma testing is in line with previous findings and also proposes that miR-486-5p may be a plasma grounded biomarker of lung cancer ${ }^{20}$. Mir-126 has been shown in human carcinogenesis as a tumor suppressor gene, and low expression has been related with breast cancer metastasis. Moreover, it has recently been found that the reduction of miR-126 expression is closely related to the clinopathological features of breast cancer ${ }^{21}$. In addition, in a study of SCC samples among miRNA profile in skin cancer patients in China, Yang et al. Mir-126 has been found to be aregulatory miRNA compared to cancer-free skin tissue. In addition, the increase in miR-126 expressed in gastric cancer cells subdued growth of cell in the G0 / G1 cell cycle phase and in vivo metastasis and carcinogenicity ${ }^{22}$. In addition, miR-126 may play a role in tumor suppression in oncogenesis efficiency by targeting the adaptive Crk protein. Our present research displays that low plasma expression of NSCLC mir-126 can differentiate patients from controls. Mir-210 can normalize the hypoxic tumor cell response and tumor growth ${ }^{23}$. High Mir-210 expression was suggestively related with estrogen and negative lymph nodes as a positive receptor for human breast cancer. Moreover, it may be one of the slowest increases in serum expression of miR-210 in diffuse large B-cell lymphoma affected patients and adenocarcinoma of pancreatic duct. Our current notes suggest that the Mir-210 may be a plasma monitor for NSCLC. Since smoking is the main reason of NSCLC, most molecular genetic changes are associated with previously diagnosed smoking with lung cancer ${ }^{24}$. The use of changes such as biomarkers can lead to over diagnosis. Interestingly, the plasma representations of the four miRNAs are independent of the smoking packets ${ }^{25}$. While our results may seem promising, this study has some limitations. Primary, as the study sample of two cohorts was very minor, and the resulting sensitivity was low for the analysis of Stage I NSCLC. There is a need to further expand the MiRNAs into independent and large cohorts' studies.

\section{CONCLUSION}

Finally, we showed that the expression of the surgically identified miRNA in cancer tumors was simple and accurate in plasma measurements. More importantly, a plasma miRNA detection panel would be used as a less invasive analytical method for NSCLC, counting long-term AC. 
However, the presence of a potential biomarker requires further verification.

\section{REFERENCES}

1. Duan, J., Shang, L., Yang, S., Wu, H., Huang, Y., \& Miao, Y (2020). miR-152/TNS1 axis promotes non-small cell lung cancer progression through Akt/mTOR/RhoA pathway. Bioscience reports.

2. Duan, J., Wang, L., Shang, L., Yang, S., Wu, H., Huang, Y., \& Miao, Y. (2021). miR-152/TNS1 axis inhibits non-small cell lung cancer progression through Akt/mTOR/RhoA pathway. Bioscience Reports, 41(1), BSR20201539.

3. Zhang, J. J., Hong, J., Ma, Y. S., Shi, Y., Zhang, D. D., Yang, X. L., ... \& Yu, F. (2021). Identified GNGT1 and NMU as Combined Diagnosis Biomarker of Non-Small-Cell Lung Cancer Utilizing Bioinformatics and Logistic Regression. Disease Markers, 2021.

4. Wang, L., Ji, X. B., Wang, L. H., Qiu, J. G., Zhou, F. M., Liu, W. J., ... \& Jiang, B. H. (2020). Regulation of microRNA-497targeting AKT2 influences tumor growth and chemoresistance to cisplatin in lung cancer. Frontiers in cell and developmental biology, 8, 840 .

5. Freitas, C., Sousa, C., Machado, F., Serino, M., Santos, V., Cruz-Martins, N., ...\&Hespanhol, V. (2021). The Role of Liquid Biopsy in Early Diagnosis of Lung Cancer. Frontiers in Oncology, 11, 1130

6. Fu, Y., Huang, L., Tang, H., \& Huang, R. (2020) hsa_circRNA_012515 is highly expressed in NSCLC patients and affects its prognosis. Cancer management and research, 12, 1877.

7. Wani, J. A., Majid, S., Khan, A., Arafah, A., Ahmad, A., Jan, B. L., ...\& Rehman, M. U. (2021). Clinico-Pathological Importance of miR-146a in Lung Cancer. Diagnostics, 11(2), 274

8. Haghjoo, N., \&Masoudi-Nejad, A. (2020). Introducing a panel for early detection of lung adenocarcinoma by using data integration of genomics, epigenomics, transcriptomics and proteomics. Experimental and molecular pathology, 112, 104360.

9. Amiri, A., Pourhanifeh, M. H., Mirzaei, H. R., Nahand, J. S., Moghoofei, M., Sahebnasagh, R., ...\& Hamblin, M. R. (2021). Exosomes and lung cancer: roles in pathophysiology, diagnosis and therapeutic applications. Current medicinal chemistry, 28(2), 308-328.

10. Uko, B. R. (2021). Deregulation of Long Non-Coding RNAs in Non-Small Cell Lung Cancer (Doctoral dissertation, The University of Liverpool (United Kingdom)).

11. Sreedurgalakshmi, K., Srikar, R., \&Rajkumari, R. (2020). CRISPR-Cas deployment in non-small cell lung cancer for target screening, validations, and discoveries. Cancer Gene Therapy, 1-15.

12. Gayosso-Gómez, L. V., \& Ortiz-Quintero, B. (2021). Circulating MicroRNAs in Blood and Other Body Fluids as Biomarkers for Diagnosis, Prognosis, and Therapy Response in Lung Cancer. Diagnostics, 11(3), 421.

13. Condrat, C. E., Thompson, D. C., Barbu, M. G., Bugnar, O L., Boboc, A., Cretoiu, D., ... \&Voinea, S. C. (2020). miRNAs as biomarkers in disease: latest findings regarding their role in diagnosis and prognosis. Cells, 9(2), 276.

14. Maheronnaghsh, M., Niktab, I., Enayati, S., Amoli, M. M., Hosseini, S. K., \&Tavakkoly-Bazzaz, J. (2021). Differentially expressed miR-152, a potential biomarker for in-stent restenosis (ISR) in peripheral blood mononuclear cells (PBMCs) of coronary artery disease (CAD) patients. Nutrition, Metabolism and Cardiovascular Diseases, 31(4), 1137-1147.

15. Jouida, A., McCarthy, C., Fabre, A., \& Keane, M. P. (2021). Exosomes: a new perspective in EGFR-mutated lung cancer. Cancer and Metastasis Reviews, 1-13.

16. Masoudi-Khoram, N., Abdolmaleki, P., Hosseinkhan, N., Nikoofar, A., Mowla, S. J., Monfared, H., \&Baldassarre, G. (2020). Differential miRNAs expression pattern of irradiated breast cancer cell lines is correlated with radiation sensitivity. Scientific reports, 10(1), 1-12.

17. Li, S., Yi, M., Dong, B., Tan, X., Luo, S., \& Wu, K. (2021). The role of exosomes in liquid biopsy for cancer diagnosis and prognosis prediction. International journal of cancer, 148(11), 2640-2651.

18. Wen, Z., Huang, Y., Ling, Z., Chen, J., Wei, X., Su, R., ...\& $\mathrm{Hu}, \mathrm{Z}$. Lack of Efficacy of Combined Carbohydrate Antigen Markers for Lung Cancer Diagnosis. Disease Markers, 2020.

19. Zou, X., Zhu, D., Zhang, H., Zhang, S., Zhou, X., He, X., ...\& Zhu, W. (2020). MicroRNA expression profiling analysis in serum for nasopharyngeal carcinoma diagnosis. Gene, 727, 144243.

20. Li, H., Zhao, J., Jia, X., Zhang, Y., Du, Y., Li, H., ...\& Huang J. A. (2020). miR-21 promotes growth, invasion and migration of lung cancer cells by AKT/P-AKT/cleavedcaspase 3/MMP-2/MMP-9 signaling pathway. International journal of clinical and experimental pathology, 13(4), 692.

21. Quu, J., Xu, J., Zhang, K., Gu, W., Nie, L., Wang, G., \& Luo, $Y$. (2020). Refining cancer management using integrated liquid biopsy. Theranostics, 10(5), 2374.

22. Malik, P., Hoidal, J. R., \& Mukherjee, T. K. (2021). Recent Advances in Curcumin Treated Non-Small Cell Lung Cancers: An Impetus of Pleiotropic Traits and Nanocarrier Aided Delivery. Current Medicinal Chemistry.

23. Tsamis, K. I., Sakkas, H., Giannakis, A., Ryu, H. S. Gartzonika, C., \&Nikas, I. P. (2021). Evaluating Infectious, Neoplastic, Immunological, and Degenerative Diseases of the Central Nervous System with Cerebrospinal Fluid-Based Next-Generation Sequencing. Molecular Diagnosis \& Therapy, 1-23.

24. Ray, S. K., \& Mukherjee, S. (2021). Cell free DNA as an evolving liquid biopsy biomarker for initial diagnosis and therapeutic nursing in Cancer-An evolving aspect in Medical Biotechnology. Current Pharmaceutical Biotechnology.

25. Huang, G., Li, X., Chen, Z., Wang, J., Zhang, C., Chen, X., ...\& Ni, L. (2020). A Three-microRNA panel in serum: serving as a potential diagnostic biomarker for renal cell carcinoma. Pathology \& Oncology Research, 26(4), 24252434. 